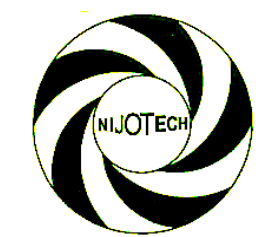

Nigerian Journal of Technology (NIJOTECH)

Vol. 39, No. 1, January 2020, pp. 212 - 218

Copyright@ Faculty of Engineering, University of Nigeria, Nsukka,

Print ISSN: 0331-8443, Electronic ISSN: 2467-8821

www.nijotech.com

http://dx.doi.org/10.4314/njt.v39i1.24

\title{
SINGLE USER TCP DOWNSTREAM THROUGHPUT PROBABILITY MODELS IN IEEE802.11B WLAN SYSTEM
}

\author{
I. Oghogho* \\ 1, Dept. of Electrical/Electronic EngR'G, Delta State Univ., Oleh Campus, Delta State, Nigeria \\ E-mail address: oghogho.ikponmwosa@delsu.edu.ng
}

\begin{abstract}
Single User, Transmission Control Protocol Downstream Throughput (TCPDST) probability models in an IEEE802.11b WLAN have been developed, validated and evaluated for performance. Measurement of single user TCPDST were taken using Tamosoft throughput test while that of signal to noise ratio (SNR) were taken using inSSIDer 2.1. The Tamosoft throughput tests were conducted using different quality of service (QOS) traffic. These QOS traffic (which were sent through an infrastructure based network) correspond to different wireless multimedia tags. Measurements were taken in free space, small offices and open corridor environments. By assuming a normal distribution, single user TCPDST Cumulative distribution function (CDF) probability models were developed for different signal categories namely: (i) all the SNR considered, (ii) strong signals only, (iii) grey signals only and (iv) weak signals only. The models were validated and their performances evaluated using root mean square (RMS) errors. RMS errors were computed by comparing model predicted values with validation data. The RMS errors for single user CDF all signals model was $0.1466 \%$. RMS errors for strong signals models, grey signals model and weak signals model respectively were $0.1466 \%, 0.6756 \%$ and $0.1233 \%$ indicating acceptable performances. All signals, strong signals, grey signals and weak signals CDF probability models predicted probabilities of obtaining TCPDST values greater than 5Mbps as $74.79 \%, 90.55 \%, 13.00 \%$ and $4.77 \%$ respectively while probabilities of obtaining TCPDST values less than 2 Mbps were predicted as 4.91\%, $0.00 \%, 18.98 \%$ and $52.41 \%$ respectively. These probability models will provide additional useful information needed to design efficient distributed data networks.
\end{abstract}

Keywords: Throughput, TCP, WLAN, probability models

\section{INTRODUCTION}

The world is presently experiencing an increasing need for wireless technologies and associated bandwidth for internet access. In many organizations, the trend is to be able to access the internet in a fast and convenient way. This has resulted due to the disruptive emergence of numerous innovative digital communication application platforms like e-governance, e-health, elearning, e-democracy, e-agriculture, etc. [1 - 3].

On the internet, network devices use network protocols to send and receive data. A network protocol defines rules and conventions for communication between network devices. Messages are sent and received as packets by protocols for computer networks using methods of packet switching. The internet protocol (IP) family contains a set of network protocols. Apart from IP, higher-level protocols like transmission control protocol (TCP), user datagram protocol (UDP), hypertext transfer protocol (HTTP) and file transfer protocol (FTP) all combine with IP to provide improved functions. Lower-level internet protocols like address resolution protocol (ARP) and internet control message protocol (ICMP) also co-exist with IP. TCP and UDP which are higher level protocols interact more closely with web browser applications while lower-level protocols interact with network adapters and other computer hardware.

Majority of network measurements are carried out using either TCP or UDP packets because most of the traffic on the internet are made of these two

\footnotetext{
* Corresponding author, tel: +234-806-067-6748
} 
protocols. TCP ensures that packets that are sent from the source do not just arrive at their destination but must do so in the order in which they were transmitted. TCP uses an algorithm that attempts to avoid network congestion by delaying the transmission of packets if it senses congestion in the network. A disadvantage of TCP compared with UDP is that it has a higher overhead than UDP. Internet control message protocol (ICMP) is used by many network engineers to design their distributed data networks even though the protocol is not a good representation of protocols like TCP and UDP usually employed in real networks $[4,5]$. An example of ICMP used in practice is the ping utility. Ping uses ICMP to probe the round trip time and responses of remote hosts of the probe messages [4].

Although network Engineers keep using ICMP to estimate the throughput between clients and servers, the throughput measurement provided by ICMP is a rough estimate of the actual throughput and errors are introduced into the estimate they provide compared with real life data. Hence being able to estimate the network throughput using TCP measurements gives a more accurate and reliable throughput data to the network engineers or designers. Wireless local area networks (WLANs) use TCP to access the internet. According to [6] IEEE802.11b WLAN system remains the most widely deployed technology that also provides the widest range although it is an old technology. Other technologies are forced to use lower data speeds when an IEEE802.11b device is introduced into the network hence in applications where an IEEE802.11b device will be introduced into the network and where the distance covered rather than high throughput or bandwidth is desired; IEEE802.11b is preferred above newer technologies [6].

Moltchanov [7] and Loiuseau, et. al [8] postulated that over $80 \%$ of the total traffic in the Internet use TCP. Tools that predict TCP performance are therefore in high demand to be able to better understand the performances of systems like WLANs which access the internet using TCP.

To characterize the performances of WLANs, the major parameters needed to be used are the throughput and round trip time (RTT) experienced by the users. Throughput indicates the average rate with which data (in bits) can be sent between one user and another in a network [9]. Upstream throughput is the speed of data sent from the client to the WLAN radio while downstream throughput is the speed of data sent from WLAN radio to the client. Upstream and downstream throughput should be studied separately because they show appreciably different characteristics $[10,11]$. Most internet users do more downloads than uploads hence specifically developing measurement tools for TCP downstream throughput is very useful today.

Several models that predict TCP throughput directly from SNR exist. Several researchers [9-22] have modelled throughput in IEEE802.11 WLANs but none of them focused on modelling the probability of obtaining a throughput value falling into a certain range for different categories of received signals. Some stochastic models [23-28] focused on packet loss probability, packet delay, back off duration, attempt and transmission probabilities, etc. but did not consider the probability of the observed TCP throughput falling within a defined throughput range for different categories of signals. Oghogho, et. al [29] provided TCP probability models for predicting upstream throughput but did not provide TCP downstream probability models. This paper by providing TCP probability models for predicting downstream throughput provides an empirical probability tool for filling this gap.

\section{RESEARCH METHOD}

The method used in this work was earlier used by [19]. However probability models were developed in this work as was done in [29]. The types of QoS traffic used in this research are described in [30]. Tamosoft throughput test was used to collect single user TCPDST field data. The received signal strength indication (RSSI) was measured in $\mathrm{dBm}$ using inSSIDer 2.1 software. inSSIDer 2.1 was also used to monitor any interfering access points. This information was used to make the decision of the most suitable channel for measurement. From the Client or server, the noise floor level was observed by logging into the WLAN radio. SNR was computed by subtracting the noise floor level from the RSSI value observed. TCPDST was measured using Tamosoft throughput Test software for each RSSI observed at different distances from the WLAN radio. The collected single user TCPDST field data were sorted into four categories using the received SNR. Single user TCPDST CDF probability models were developed for predicting the probability of single user TCPDST falling into different throughput ranges for various SNRs. From all data collected, one model which relates single user TCPDST with SNR categories was 
developed. One model was developed for all signals considered another was developed for Strong signals only (SNR $>25 \mathrm{~dB}$ ), another model was developed for grey signals only $(25 \mathrm{~dB}>\mathrm{SNR}>18 \mathrm{~dB})$ and yet another model was developed for weak signals only (SNR $<19 \mathrm{~dB})$. The models were compared with the validation data collected in different but similar environments to that where the field data were collected. RMS errors for the developed models were computed for all signal categories to check the validity of the models.

\section{RESULTS AND DISCUSSION}

Table 1 shows field data and Validation data statistical Parameter values of Single user TCPDST for different categories of SNR. From the Table, both the field and the validation data compare well for the different statistical parameters considered even though the measurements were taken in different similar environments. To develop the models, a normal distribution was assumed to derive a general function for predicting the probability distribution function (PDF) of obtaining TCPDST values for the different categories of SNR. This was also extended to develop Cumulative distribution function (CDF) models of obtaining TCPDST values for all SNR, Strong signals only, Grey signals only and Weak signals only. The normal or Gaussian distribution is defined by the mathematical model [31].

$$
\mathrm{f}(\mathrm{x})=\frac{1}{\sigma \sqrt{2 \pi}} \mathrm{e}^{-\frac{1(x-\mu)^{2}}{2} \sigma^{2}(-\infty<x<+\infty)}
$$

Where $\sigma=$ standard deviation of the population, $\mu$ is the population mean, and $\mathrm{x}$ is the downstream Throughput being investigated. $f(x)$ is the PDF of obtaining a TCPDST $=x$ Mbps. From Table 1 , the required standard deviations and mean values are obtained and inserted into equation 1 to obtain the PDF models or equations for the normal distribution assumed in the various categories of signals for a single user on the network. Since throughput cannot be negative, the range is from 0 to infinity. The PDFs have little practical importance because they estimate the probability of obtaining a particular TCPDST value and not a range of TCPDST values.

This leads to the need to evaluate the CDF in terms of standard units. To obtain CDF in terms of standard units, equation 1 is transformed to the standard normal form shown in equation 2 [31].

$$
\mathrm{f}(\mathrm{Z})=\frac{1}{\sigma \sqrt{2 \pi}} \mathrm{e}^{-\frac{1(\mathrm{Z})^{2}}{2 \sigma^{2}}(-\infty<x<+\infty)}
$$

Where

$$
Z=\frac{\mathrm{x}-\mu}{\sigma}
$$

and $\sigma=$ standard deviation of the population and $\mu$ is the population mean. For a single user on the network, $Z$ scores or values can be obtained using model equations 4, 5, 6, and 7 for all SNR, strong signals, grey signals and weak signals respectively.

$$
\begin{array}{ll}
Z=\left(\frac{x-5.232}{1.3374}\right)+\gamma & (0 \leq x \leq \infty) \\
Z=\left(\frac{x-5.749}{0.6044}\right)+\gamma \quad(0 \leq x \leq \infty) \\
Z=\left(\frac{x-3.392}{1.3702}\right)+\gamma \quad(0 \leq x \leq \infty) \\
Z=\left(\frac{x-2.1143}{1.5196}\right)+\gamma(0 \leq x \leq \infty)
\end{array}
$$

$\boldsymbol{\gamma}$ is the correction factor that was introduced to make the models more accurate. The CDF is given by equation 8 and for any value of $Z$ score in equations $4,5,6$, and 7 , the CDF value can be found from the standard normal distribution table [31].

$$
F(Z)=\frac{1}{\sqrt{2 \pi}} \int_{-\infty}^{Z} \mathrm{e}^{-\frac{1}{2} \mathrm{u}^{2}} \mathrm{du}
$$

The correction factor $(\boldsymbol{\gamma})$ was computed by comparing the values from the CDF probability models with the field data probabilities of obtaining TCPDST in the various SNR categories. Thus after the CDF probabilities are estimated using the respective models from $Z$ tables, the correction factors are obtained by subtracting the model values from $Z$ tables from the field data probabilities. Table 2 shows the unadjusted $C D F$ probability models values obtained using $Z$ tables, the adjusted TCPDST CDF probability models values and the correction factors introduced to make them more accurate in predicting the probability of obtaining TCPDST for different signal categories. 
Table 1: Values of Statistical Parameters of Single user TCPDST for different SNR.

\begin{tabular}{|c|c|c|c|c|}
\hline \multirow{2}{*}{$\begin{array}{l}\text { Statistical } \\
\text { Parameter }\end{array}$} & \multicolumn{2}{|c|}{$\begin{array}{l}\text { ALL RSSI (SNR) considered (63dB } \geq \\
\text { SNR } \geq \mathbf{1 3 d B})\end{array}$} & \multicolumn{2}{|c|}{ Strong Signal (SNR $\geq 25 \mathrm{~dB}$ ) } \\
\hline & $\begin{array}{l}\text { TCPDST Field data } \\
\text { (Mbps) }\end{array}$ & $\begin{array}{l}\text { TCPDST Validation } \\
\text { data }\end{array}$ & $\begin{array}{l}\text { TCPDST Field data } \\
\text { (Mbps) }\end{array}$ & TCPDST Validation data \\
\hline $\mathrm{N}$ (Sample Size) & 1885 & 762 & 1506 & 568 \\
\hline Mean & 5.232 & 4.975 & 5.749 & 5.591 \\
\hline Std. Error of Mean & 0.0308 & 0.0526 & 0.0156 & 0.0302 \\
\hline Median & 5.85 & 5.7050 & 5.94 & 5.8900 \\
\hline Mode & 6.03 & $5.92^{*}$ and $6.08^{*}$ & 6.03 & $5.92^{*}$ and $6.08^{*}$ \\
\hline Std. Deviation & 1.3374 & 1.4512 & 0.6044 & 0.7192 \\
\hline Variance & 1.788 & 2.106 & 0.365 & 0.517 \\
\hline $\begin{array}{l}\text { Coefficient of } \\
\text { dispersion }\end{array}$ & 0.2556 & 0.29172 & 0.1051 & 0.128628 \\
\hline Skewness & -1.698 & -1.513 & -1.747 & -1.902 \\
\hline $\begin{array}{l}\text { Std. Error of } \\
\text { Skewness }\end{array}$ & 0.056 & 0.089 & 0.063 & 0.103 \\
\hline Kurtosis & 2.469 & 1.494 & 7.106 & 4.254 \\
\hline $\begin{array}{l}\text { Std. Error of } \\
\text { Kurtosis }\end{array}$ & 0.113 & 0.177 & 0.126 & 0.205 \\
\hline Range & 10 & 7.13 & 7.65 & 6.18 \\
\hline $\begin{array}{l}\text { Statistical } \\
\text { Parameter }\end{array}$ & \multicolumn{2}{|c|}{ Grey signal $(25 \mathrm{~dB}>\mathrm{SNR} \geq 19 \mathrm{~dB})$} & \multicolumn{2}{|l|}{ Weak Signal (SNR<19dB) } \\
\hline N (Sample Size) & 316 & 98 & 63 & 95 \\
\hline Mean & 3.3915 & 4.0413 & 2.1143 & 2.2459 \\
\hline Std. Error of Mean & 0.07708 & 0.11572 & 0.19145 & 0.13923 \\
\hline Median & 3.2750 & 4.3500 & 1.91 & 2.1000 \\
\hline Mode & $\begin{array}{l}2.76^{*}, 3.2^{*} \text { and } \\
4.89^{*}\end{array}$ & 5.01 & $\begin{array}{l}0.36^{*} 0.54^{*} 0.56^{*} 1.15^{*} \\
2.56^{*} 3.1^{*}\end{array}$ & $\begin{array}{l}3.73^{\star}, 1.87^{\star}, 0.77^{\star}, 0.74^{*}, \\
0.67^{\star}, 0.39^{*}\end{array}$ \\
\hline Std. Deviation & 1.3702 & 1.14553 & 1.5196 & 1.35703 \\
\hline Variance & 1.878 & 1.312 & 2.309 & 1.842 \\
\hline $\begin{array}{l}\text { Coefficient of } \\
\text { dispersion }\end{array}$ & 0.4041 & 0.2834558 & 0.7187 & 0.6042255 \\
\hline Skewness & 0.144 & -0.551 & 1.159 & 0.335 \\
\hline $\begin{array}{l}\text { Std. Error of } \\
\text { Skewness }\end{array}$ & 0.137 & 0.244 & 0.302 & 0.247 \\
\hline Kurtosis & 0.188 & -0.324 & 1.874 & -1.085 \\
\hline $\begin{array}{l}\text { Std. Error of } \\
\text { Kurtosis }\end{array}$ & 0.273 & 0.483 & 0.595 & 0.490 \\
\hline Range & 9.19 & 5.14 & 7.47 & 4.85 \\
\hline
\end{tabular}

${ }^{*}$ multiple mode exists

Table 2: TCPDST CDF probability models values

\begin{tabular}{|c|c|c|c|c|c|c|}
\hline \multicolumn{4}{|c|}{ ALL RSSI (SNR) considered (63dB $\geq S N R \geq 13 \mathrm{~dB})$} & \multicolumn{3}{|c|}{ Strong Signals (SNR $\geq 25 \mathrm{~dB}$ ) } \\
\hline $\begin{array}{l}\text { TCPDST } \\
\text { (Mbps) }\end{array}$ & $\begin{array}{l}\text { CDF probability } \\
\text { model value } \\
\text { from Z table }\end{array}$ & $\begin{array}{l}\text { Correction } \\
\text { factor }(\gamma)\end{array}$ & $\begin{array}{l}\text { Adjusted CDF } \\
\text { Probability } \\
\text { Model values }\end{array}$ & $\begin{array}{l}\text { CDF probability } \\
\text { model value } \\
\text { from Z table }\end{array}$ & $\begin{array}{l}\text { Correction } \\
\text { factor }(\gamma)\end{array}$ & $\begin{array}{l}\text { Adjusted CDF } \\
\text { Probability } \\
\text { Model values }\end{array}$ \\
\hline$>6$ & 0.3121 & 0.013 & 0.3251 & 0.3372 & 0.067 & 0.4042 \\
\hline $5-5.99$ & 0.2118 & 0.211 & 0.4228 & 0.5553 & -0.054 & 0.5013 \\
\hline $4-4.99$ & 0.2052 & -0.111 & 0.0942 & 0.1056 & -0.04 & 0.0656 \\
\hline 3-3.99 & 0.1479 & -0.075 & 0.0729 & 0.0019 & 0.026 & 0.0279 \\
\hline $2-2.99$ & 0.0794 & -0.042 & 0.0374 & 0.0000 & 0.001 & 0.0010 \\
\hline $1-1.99$ & 0.0317 & 0.002 & 0.0337 & 0.0000 & 0 & 0.0000 \\
\hline$<0.99$ & 0.0094 & 0.006 & 0.0154 & 0.0000 & 0 & 0.0000 \\
\hline \multicolumn{7}{|c|}{ Grey signals $(25 \mathrm{~dB}>\mathrm{SNR} \geq 19 \mathrm{~dB})$} \\
\hline$>6$ & 0.0287 & -0.022 & 0.0067 & 0.0052 & 0.011 & 0.0162 \\
\hline $5-5.99$ & 0.0923 & 0.031 & 0.1233 & 0.0235 & 0.008 & 0.0315 \\
\hline $4-4.99$ & 0.2090 & 0.019 & 0.2280 & 0.0788 & 0.016 & 0.0948 \\
\hline 3-3.99 & 0.2841 & -0.006 & 0.2781 & 0.1735 & -0.062 & 0.1115 \\
\hline
\end{tabular}




\begin{tabular}{lllllll}
\hline ALL RSSI (SNR) considered & $(63 \mathrm{~dB} \geq \mathrm{SNR} \geq 13 \mathrm{~dB})$ & \multicolumn{3}{l}{ Strong Signals $(\mathrm{SNR} \geq 25 \mathrm{~dB})$} & \\
\hline TCPDST & $\begin{array}{l}\text { CDF probability } \\
\text { (Mbps) }\end{array}$ & $\begin{array}{l}\text { Correction } \\
\text { from value }\end{array}$ & $\begin{array}{l}\text { Adjusted CDF } \\
\text { factor }(\gamma) \\
\text { Probability } \\
\text { Model values }\end{array}$ & $\begin{array}{l}\text { CDF probability } \\
\text { model value } \\
\text { from Z table }\end{array}$ & $\begin{array}{l}\text { Correction } \\
\text { factor }(\gamma)\end{array}$ & $\begin{array}{l}\text { Adjusted CDF } \\
\text { Probability } \\
\text { Model values }\end{array}$ \\
\hline $2-2.99$ & 0.2320 & -0.058 & 0.1740 & 0.2509 & -0.029 & 0.2219 \\
$1-1.99$ & 0.1138 & 0.044 & 0.1578 & 0.2354 & -0.013 & 0.2224 \\
$<0.99$ & 0.0460 & -0.014 & 0.0320 & 0.2327 & 0.069 & 0.3017 \\
\hline
\end{tabular}

Table 3: RMS errors for Adjusted CDF Probability Model for TCPDST.

\begin{tabular}{lllll}
\hline Signal Category & All SNR & Strong Signals & Grey Signals & Weak Signals \\
\hline RMS Error & 0.0015 & 0.0015 & 0.0068 & 0.0012 \\
\hline
\end{tabular}
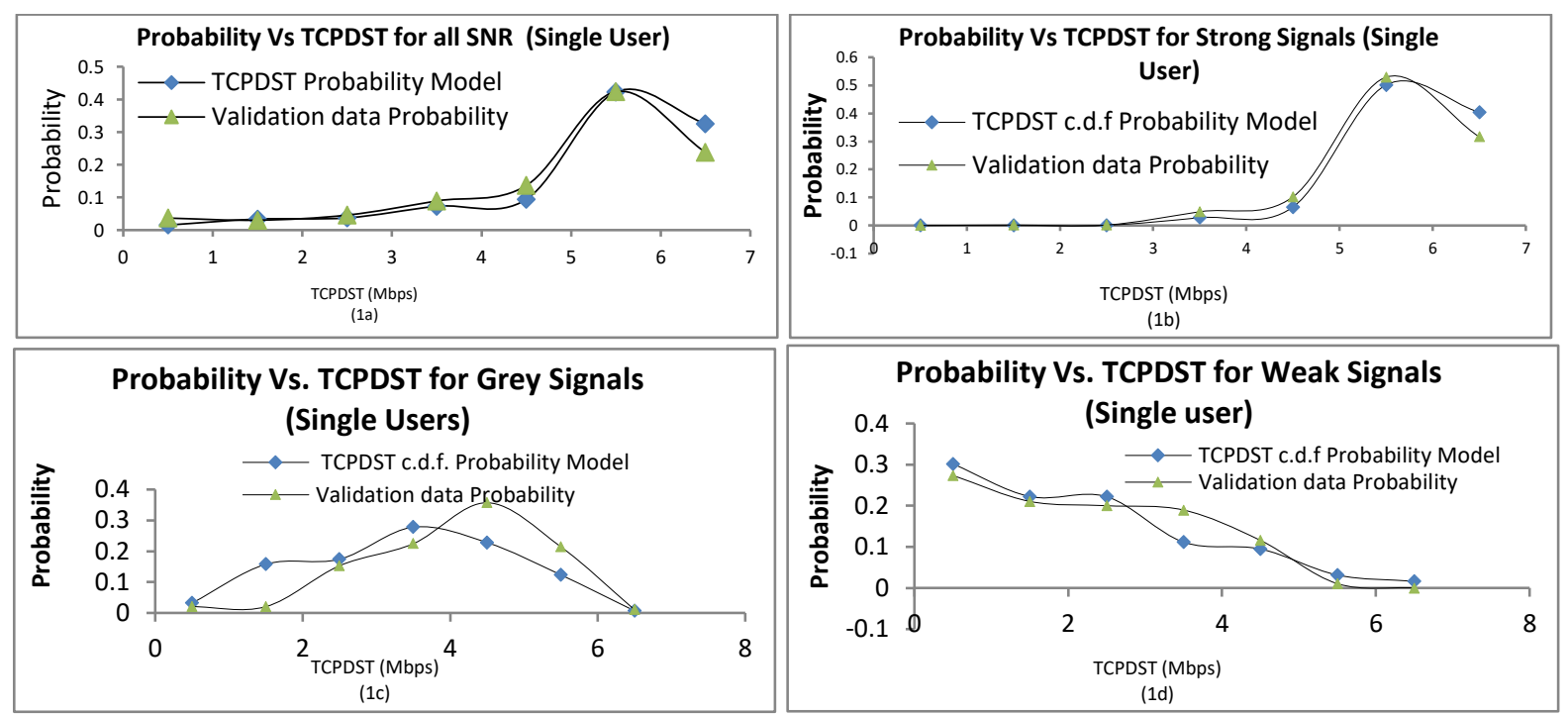

Fig. 1: Graph of Probability Vs TCPDST for developed models and Validation data.

For a single user, the models probabilities and that of validation field data are plotted against TCPDST in Figure 1. Fig.1(a) shows the graph for all SNR, Fig.1(b) shows the graph for strong signals only, Fig.1(c) shows the graph for grey signals only and Fig.1(d) show graphs for weak signals only. It can be seen from the graphs that the model predicted probabilities follow the validation data probabilities closely in all categories of signals. A bimodal distribution is observed in the grey signal range.

For a single user on the network, the all signals, strong signal, grey signal and weak signal CDF probability models respectively predicted probabilities of obtaining TCPDST values greater than $5 \mathrm{Mbps}$ as $74.79 \%, \quad 90.55 \%, \quad 13.00 \%$ and $4.77 \%$ while probabilities of obtaining TCPDST values less than 2 Mbps were predicted as $4.91 \%, 0.00 \%, 18.98 \%$ and $52.41 \%$ respectively. Thus when a signal is strong and there is a single user on the network, there is a very high probability (over $90 \%$ ) of obtaining a TCPDST value greater than $5 \mathrm{Mbps}$ and a practically near zero possibility to obtain a value less than 2Mbps. On the other hand when signal has become weak there is a high probability (52.41\%) of obtaining a TCPDST less than 2Mbps. RMS errors were computed to test the validity and performance of the developed probability models.

This was done by comparing the developed model predicted probabilities with validation data probabilities. Table 3 shows the RMS errors for the adjusted CDF probability TCPDST models in the single user scenario estimated for all SNR, strong signals only, grey signals only and weak signals only. All the models have good performance as they show low RMS errors. In the single user scenario, the adjusted CDF probability TCPDST model for grey signals showed the largest error $(0.0068$ or $0.6756 \%)$ observed which is reasonably negligible.

\section{FUTURE RESEARCH DIRECTIONS, RECOMMENDATIONS AND CONCLUSION}

In this work (by assuming a normal distribution for the collected data), empirical CDF probability models that will enable researchers and WLAN users to estimate the probability that TCPDST falls into various throughput ranges for various categories of signals 
when there is a single user on the network have been developed validated and tested for performance. All CDF probability models developed showed low RMS errors which are within acceptable limits as shown in Table 3. However, the research needs to also cover multiple users TCPDST as this is what is most experienced in real life scenarios. In addition, it is necessary to consider models developed for specific QoS traffic types and specific environments to allow network users have tools for predicting such scenarios. Probability models for newer WLAN systems such as IEEE $802.11 \mathrm{~g} / \mathrm{n}$ and LTE systems should also be considered for development since the old technologies are gradually getting out of use.

This work has formed the background for the future work identified above which are already being considered by us in our on-going research. In addition to already existing throughput prediction models, our probability models will provide some additional useful information needed to design very efficient distributed data network.

\section{ACKNOWLEDGEMENTS}

The Author acknowledges Mr Folarin Oluwafemi E. whose guide helped in the set up of the measurement testbed. Also acknowledged are Engr. Owamah $\mathrm{H}$., Mr Adejumo, Mr Ikubanni S. and Mr. Adegbesin who provided useful guide in data sorting and analysis. Dr. Osewmengie Omoefosa, Prof F.O Edeko, Engr. Egbune Dickson, Prof Emagbetere J.O, Prof J.S. Adeniyi, Dr. Gana A. J and Dr. Henry Ojongbede are also deeply acknowledged for their support.

\section{REFERENCES}

[1]. Lin, S. Wen, X. Hu, Z. \& Lu, Z. "Improving throughput through dynamically tuning contention window size in dense wireless network" The Journal of China Universities of Posts and Telecommunications, Volume 24, Issue 4, Pages 27-33, (2017)

[2]. Oghogho, I. \& Ezomo, P. I. "ICT for National Development in Nigeria: Creating an Enabling Environment" International Journal of Engineering and Applied Sciences. Vol 3 (2) pp 59-66, (2013).

[3]. Moltchanov, D. "Performance models for wireless channels" Computer Science Review Vol.4 (2010) pp153 - 184, (2010).

[4]. Mitchell, B. "ICMP". About.com Wireless/Networking. http://compnetworking.about.com/od/tcpip/g/ bldef_icmp.htmat Accessed on $12^{\text {th }}$ June 2014.

[5]. Rouse, M. "ICMP (Internet Control Message Protocol)". Search Networking TechTarget. http://searchnetworking.techtarget.com/defini tion/ICMP Accessed on 12 $2^{\text {th }}$ June 2014.

[6]. Khanduri, R. \& Rattan, S. S. "Performance Comparison Analysis Between IEEE802.11a/b/g/n Standards". International Journal of Computer Applications. Vol 78 (1) pp13-20 (2013).

[7]. Moltchanov, D. "A study of TCP performance in wireless environment using fixed-point approximation". Computer Networks Vol. 56 (2012)1263-1285 (2012).

[8]. Loiseau, P. Gonçalves, P. Barral, J. \& Primet V. P. "Modeling TCP throughput: An elaborated large-deviations-based model and its empirical validation" Performance Evaluation Vol. 67 pp1030-1043 (2010).

[9]. Henty, B. E. "Throughput Measurements and Empirical Prediction Models for IEEE 802.11b Wireless LAN (WLAN) Installations". Masters of Science in Electrical Engineering Thesis, Virginia Polytechnic Institute and State University (2001). http://scholar.lib.vt.edu/theses/available/etd08142001172523/unrestricted/hentythesis.pdf Accessed on $21^{\text {st }}$ August 2012.

[10]. Oghogho, I. Fredrick, O. E. \& Emagbetere, J. E. "Measurement and Modeling of TCP Downstream Throughput Dependence on SNR in an IEEE802.11b WLAN System". Journal of King Saud University-Engineering Sciences. Vol. (2018) 30: 170-176 (2018).

[11]. Boris, B. Katarzyna, K. "AP-initiated multi-user transmissions in IEEE 802.11ax WLANs" Ad Hoc Networks, Volume85, Pages145-159, (2019).

[12]. Kosuke, S. Nobuyoshi, K. Zhetao, L. Tingrui, P. Young-June C. Hiroo, S. "Generalized analytical expressions for end-to-end throughput of IEEE 802.11 string-topology multi-hop networks" Ad Hoc Networks, Volume 70, Pages 135-148 (2018).

[13]. Bruno, R. Conti, M. \& Gregory, E. "An accurate closed-form formula for the throughput of long-lived TCP connections in IEEE 802.11 WLANs". Computer Networks, Volume 52(1) pp199-212, (2008).

[14]. Padhye, J. Firoiu, V. Towsley, D. \& Kurose, J. "Modeling Throughput: A simple Model and its 
Empirical Validation". IEE/ACM Transactions on Networking Vol (8) pp133-145 (1998).

[15]. Gau, R. H. \& Chen, K. M. "Probability Models for the Splitting Algorithm in Wireless Access Networks with Multipacket Reception and Finite Nodes". IEEE Transactions on Mobile Computing.Vol 7 (12) pp1519-1535 (2008).

[16]. Pradeepa, B. K. \& Joy, K. "Aggregate download throughput for TCP-controlled long file transfers in a WLAN with multiple STA-AP association rates". Performance Evaluation Vol69(2012) pp289-296, (2012).

[17]. Metreaud, L.T. "An RF-Isolated Real-Time Multipath Test bed for Performance Analysis of WLANS". Masters of Science in Electrical and Computer Engineering Thesis. Worcester Polytechnic Institute. pp1-123 (2006). http://www.cwins.wpi.edu/publications/Thesis /MS\%20Thesis/leon\%20metreaud.pdf Accessed on $6^{\text {th }}$ November 2011.

[18]. Ng, B. Tan, Y. F. Tan, S. W. \& Lee. S. W. "Throughput and PER estimates harnessing link-layer measurements for indoor 802.11n WLAN". Computer Standards \& Interfaces Vol.34(2012) pp135- 145 (2012).

[19]. Oghogho, I. Edeko, F. O. Emagbetere, J. E. \& Victor, M. "Empirical investigation on the dependence of TCP upstream throughput on SNR in an IEEE802.11b WLAN system". In Telecommunications (ICT), 2014, 21st International Conference on, pp. 442-446 (2014).

[20]. Oghogho, I. Edeko, F. O. \& Emagbetere, J. E. "Investigation on the Dependence of TCP Upstream Throughput on SNR For Single and Multiple Links in a WLAN System". Review of Information Engineering and Applications, Vol 2(1), 15-32 (2015).

[21]. Oghogho, I. Fredrick, O. E. \& Emagbetere, J. E. "Empirical Investigation on the Dependence of TCP Downstream Throughput on SNR in an IEEE802.11b WLAN System". Journal of King Saud University-Engineering Sciences. Vol (2017) 29: 135-143, (2017).

[22]. Oghogho, I. "Throughput dependence on SNR in IEEE 802.11 WLAN systems" In Ed. Mehdi Khosrow-Pour, Encyclopedia of Information
Science and Technology, Fourth Edition. IGI Global USA. pp 6618-6629, (2018).

[23]. Weigle, M. C. Cheng, L. Kaur, J. \& Kulkarni, V. "Generalized Stochastic Performance Models for Loss-based Congestion Control". Computer Communications Vol 33(2010) pp513-525 (2010).

[24]. Gupta, V. Dharmaraja, S. \& Gong, M. "Analytical modelling of TCP flow in wireless LANs" Mathematical and Computer Modelling Vol53(2011) pp684-693, (2011).

[25]. Chunyu, H. Hwangnam, K. \& Jennifer, C.H. "Short-term non-uniform access in IEEE 802.11-compliant WLANs: A study on its impact on the saturation performance". Computer Networks, Volume 52, (1) 61-76, (2008).

[26]. Panda, M. K. \& Kumar, A. "State Dependent Attempt Rate modelling of single cell IEEE802.11 WLANs with homogeneous nodes and Poisson packet arrivals". Performance Evaluation Vol 69(2012) 413-439, (2012).

[27]. Min, G. Hu, J. \& Woodward, M. E. "Modeling and analysis of TXOP differentiation in infrastructure-based WLANs". Computer Networks Vol. 55 (2011) 2545-2557, (2011).

[28]. Kafetzakis, E. Kontovasilis, K. \& Stavrakakis, I. "Effective-capacity-based stochastic delay guarantees for systems with time-varying servers, with an application to IEEE802.11 WLANs". Performance Evaluation Vol. 68 (2011) 614-628, (2011).

[29]. Oghogho, I. Edeko, F. O. \& Emagbetere, J. E. "Probability Models for Predicting TCP Upstream Throughput in an IEEE802.11b WLAN System". Journal of Electronic and Electrical Engineering, 12(2), 1-19, (2015).

[30]. www.tamos.com "Throughput test". www.tamos.com. www.tamos.com $/ \mathrm{htm} / \mathrm{help} / \mathrm{tt} /$ performing_the tests.htm. Accessed on 9th April 2019.

[31]. Ify, L. N. "Probability and Statistics for Science and Engineering Practice". University of Port Harcourt Press (2011). 